\title{
Extracción de compuestos fitoquímicos de inflorescencia y frutos de guanábana (Annona muricata L.)
}

Extraction of phytochemical compounds from soursop inflorescence and fruit (Annona muricata L.)

\author{
Andrés Eloy León-Fernández ${ }^{1}$ (D) , Rosendo Balois-Morales ${ }^{2 *}$ (D) , Pedro Ulises Bautista-Rosales ${ }^{2}$ (D) , \\ Yolotzin Apatzingan Palomino-Hermosillo ${ }^{2}$ (D), \\ Juan Esteban Bello-Lara ${ }^{1}$ (D) , Consuelo Esperanza López-Rivas ${ }^{3}$
}

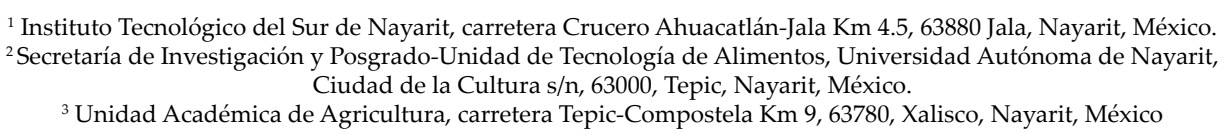

* Autor para correspondencia: balois_uanayar@hotmail.com

\section{Fecha de recepción:}

11 de febrero de 2021

Fecha de aceptación:

13 de agosto de 2021

Disponible en línea:

21 de diciembre de 2021

Este es un artículo en acceso abierto que se distribuye de acuerdo a los términos de la licencia Creative Commons.

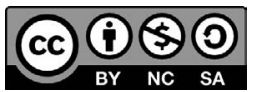

Reconocimiento-

NoComercia-

CompartirIgual 4.0

Internacional

\section{RESUMEN}

En guanábana (Annona muricata L.) se han identificado 212 compuestos bioactivos, entre los cuales destacan las acetogeninas, los alcaloides y los fenólicos, cuya presencia o concentración varía en cada órgano de A. muricata durante el ciclo de vida. El objetivo de este estudio fue determinar un método de extracción de compuestos bioactivos (terpenoides, esteroides, alcaloides, acetogeninas, compuestos fenólicos y saponinas) en órganos estructurales que componen la inflorescencia y fruto de guanábana, utilizando solventes orgánicos. Se usaron inflorescencias y frutos de guanábana durante su crecimiento y desarrollo con una longitud de: 2, 5-7, 8-10, 13-15, 17-23 y 25-30 cm, para evaluar dos métodos de extracción (sonicación y macerado) y cuatro solventes (hexano, cloroformo, etanol y metanol). Se determinó la presencia y concentración de compuestos bioactivos en inflorescencia, epidermis y pulpa. Mediante sonicado y con metanol se obtuvo la mayor extracción de compuestos bioactivos. Las concentraciones más elevadas de compuestos fenólicos se encontraron en inflorescencia y epidermis de los frutos; de flavonoides en inflorescencia, y alcaloides y flavonoides en la pulpa.

\section{PALABRAS CLAVE}

Sonicado, alcaloides, fenoles totales, flavonoides, solvente.

\section{AbSTRACT}

In soursop (Annona muricata L.), 212 bioactive compounds have been identified, highlighting acetogenins, alkaloids and phenolics, whose presence or concentration varies in each organ of $A$. muricata during its life cycle. The objective of this study was to determine a method of extraction of bioactive compounds (terpenoids, steroids, alkaloids, acetogenins, phenolic compounds and saponins) in structural organs that make up the soursop inflorescence and fruit, using organic solvents. Soursop inflorescences and fruits were used during its growth and development at a length of: 2, 5-7, 8-10, 13-15, 17-23, and 25-30 cm, evaluating two extraction methods (sonication and mashing), and four solvents (hexane, chloroform, ethane, and methanol). The presence and concentration of bioactive compounds in inflorescence, epidermis and pulp were determined. The highest extraction of compounds was obtained by means of methanol and sonication. The highest concentrations of phenolic compounds were found in inflorescences and fruit epidermis, flavonoids were found in inflorescence, and alkaloids and flavonoids in pulp.

\section{KEYWORDS}

Sonicated, alkaloids, total phenols, flavonoids, solvent. 


\section{INTRODUCCIÓN}

Los metabolitos secundarios o productos naturales son sintetizados en pequeñas cantidades por las plantas a través del carbono asimilado y de la energía proveniente de la fotosíntesis; su producción se restringe a ciertos géneros, familias y especies de plantas (Ávalos-García y Pérez-Urria 2009). Los metabolitos secundarios, tales como los alcaloides, fenoles, flavonoides y taninos, funcionan como atrayentes, repelentes, protectores, tóxicos, plaguicidas, pigmentos, entre otros, lo que les confiere valor medicinal, farmacéutico, cosmético y alimentario (Cabrera-Carrión et al. 2017), pero no actúan en el metabolismo primario.

Los factores que influyen en la fluctuación de la presencia y concentración de metabolitos secundarios se pueden dividir en: genéticos, ontogéneticos, morfogéneticos y factores ambientales (Verma y Shukla 2015). Todo el proceso para transportar y almacenar metabolitos secundarios proviene de la vía biosintética y éstos son influenciados por factores químicos y celulares que se relacionan con factores abióticos y del desarrollo de la planta (Broun et al. 2006).

Tradicionalmente, la guanábana, Annona muricata L., ha sido utilizada para fines medicinales (Coria-Téllez et al. 2018), sin tener un conocimiento científico de los compuestos bioquímicos que se encuentran en los diferentes órganos de la planta, por ejemplo, hojas, tallo, raíz, semilla y epicarpio, lo que ha motivado la evaluación de las actividades biológicas en un gran número de extractos. Se ha reportado que existen hasta 212 compuestos bioactivos en A. muricata (Moghadamtousi et al. 2015). Uno de los principales compuestos bioactivos que han llamado la atención son las acetogeninas (ACGs) (Badrie y Schauss 2010; Cordeiro y Pinto 2005), también los alcaloides y compuestos fenólicos (Jimenez et al. 2014; Yang et al. 2015).

Las acetogeninas son una serie de derivados de los policétidos y de ácidos grasos que poseen anillos de tetrahidrofurano y una $\gamma$-lactona metilada (a veces reorganizada a una metilcetolactona), los cuales, con varios grupos hidroxilo, acetoxilo y ketoxilo, a lo largo de la cadena de hidrocarburos, son solubles en disolventes orgánicos (Rupprecht et al. 1990). Para determinar las características estructurales que diferencian a las acetogeninas, es necesario llevar a cabo un asilamiento y purificación de las mismas; la mayoría de los fraccionamientos de acetogeninas se realizan empleando disolventes orgánicos de diferente polaridad (Agu et al. 2018; Hernández-Fuentes et al. 2019). No existen estudios científicos que reporten la identificación de acetogeninas en pulpa de guanábana (Champy et al. 2005; Melot et al. 2009; Sun et al. 2014) Generalmente, se describen grupos de acetogeninas que presentan isomerías estructurales y dificultan su separación, dentro de las cuales se encuentran isómeros de acetogeninas monofuránicas (Sun et al. 2014). En la pulpa de guanábana se encuentran alcaloides tales como anonaina, asimilobina, N-metilcoculaurina nomurciferina y reticulina, cuya característica principal es la presencia de átomos de nitrógeno en su estructura química (Hasrat et al. 1997a; Hasrat et al. 1997b; Kotake et al. 2004). Por otro lado, estos alcaloides reportados son el tipo isoquinolina, aporfina y protoberberina (Mohanty et al. 2008).

Los compuestos fenólicos son moléculas que se caracterizan por tener anillos aromáticos o bencénicos, $y$, unidos a éstos, se encuentran insaturaciones como grupos OH (Abarca-Vargas y Petricevich 2018). Los compuestos fenólicos más importantes encontrados en guanábana incluyen quercentina (Nawwar et al. 2012) y el ácido gálico (Correa-Gordillo et al. 2012). De igual forma, se reporta la presencia de 37 compuestos fenólicos; flavonoides, y antioxidantes lipofílicos, como tocoferoles.

El objetivo de este estudio fue determinar un método de extracción de compuestos bioactivos (terpenoides, esteroides, alcaloides, acetogeninas, compuestos fenólicos y saponinas) en órganos estructurales que componen el fruto de guanábana, utilizando un solvente orgánico.

\section{Materiales y Métodos}

La recolección de material vegetal se realizó de octubre de 2017 a febrero de 2018, cada mes, hasta madurez fisiológica, y a los 10 días, para madurez de consumo, en el Ejido de Altavista, Municipio de Compostela, Nayarit, México $\left(21^{\circ} 04^{\prime} 28^{\prime \prime} \mathrm{N}\right.$ y $105^{\circ} 24^{\prime} 56^{\prime \prime} \mathrm{O}, 21$ msnm), con precipitación media anual de 1,258 mm, y temperatura media anual de $22.9{ }^{\circ} \mathrm{C}$ (CONAGUA 
2017; INEGI 2017). Los huertos no tienen un manejo agronómico establecido, debido a que el productor no lo considera como una prioridad. Los árboles de estos huertos tienen una edad de 30 años, y son plantas criollas propagadas por semilla.

El material vegetal fueron inflorescencias y frutos de guanábana; estos últimos se clasificaron por longitud, desde los 2 (forma de "cepillo") hasta los 30 cm (madurez de consumo) en el árbol; a los frutos se les separó la epidermis y la pulpa, con la finalidad de determinar los compuestos fitoquímicos por separado. Así, durante el crecimiento y desarrollo del fruto, las etapas fenológicas se clasificaron por longitud: 2, 5 a 7 , 8 a 10, 13 a 15, 17 a $23 \mathrm{~cm}$ (madurez fisiológica) y 25-30 $\mathrm{cm}$ (madurez de consumo), tomando como referencia el índice de cosecha reportado por Jiménez-Zurita et al. (2016), que son 160 días después de la antesis. El material vegetal fue llevado al laboratorio de análisis especiales de la unidad de tecnología de alimentos, donde fue lavado, empaquetado y almacenado a $-20{ }^{\circ} \mathrm{C}$ (congelador TORREY ${ }^{\odot}$, modelo CHTC16OW A, Utah, USA) hasta su uso. Posteriormente, se deshidrataron en un liofilizador $\left(\mathrm{LABCONCO}^{\circ}\right.$, mod. 2.5, Kansas City, Missouri, USA).

Esta investigación se llevó a cabo en dos fases: la primera se realizó de manera preliminar para obtener datos de presencia (+) y ausencia (-) de los metabolitos en inflorescencia, y para establecer las etapas fenológicas del crecimiento y desarrollo de fruto de guanábana, que fueron sometidos a extracción por dos métodos (macerado y sonicado), y cuatro solventes de extracción (hexano, cloroformo, etanol y metanol). Al finalizar esta primera fase, se seleccionó el mejor solvente (metanol), con mayor presencia de compuestos fitoquímicos.

Para la segunda fase, se continuaron utilizando los dos métodos de extracción de la primera fase, y el metanol como solvente. En esta fase, el diseño experimental fue completamente al azar en arreglo factorial $(2 \times 2 \times 7)$, esto es, dos métodos de extracción, dos componentes del fruto (pulpa y epidermis) y siete etapas fenológicas del crecimiento y desarrollo del fruto. Se utilizó una prueba de comparación de medias de DMS (Diferencia Mínima Significativa) cuando el ANOVA mostró diferencias estadísticamente significativas $(P<0.05)$. Los análisis estadísticos fueron realizados con el software STATISTICA versión 10 .

\section{Extracción de compuestos fitoquímicos}

Una vez liofilizados las inflorescencias y los frutos para el estudio, éstos se trituraron en un molino (KRUPS@ modelo F408, Solingen, Alemania) hasta obtener un polvo homogéneo. Para la extracción de los compuestos fitoquímicos se pesaron $2 \mathrm{~g}$ de material liofilizado y se depositaron en un matraz Erlenmeyer de $50 \mathrm{~mL}$; a éste se le agregaron $20 \mathrm{~mL}$ de disolventes orgánicos (hexano, cloroformo, etanol y metanol). La extracción se llevó a cabo a través de los métodos de macerado con agitación y sonicación (León et al. 2017).

La extracción por macerado se realizó en una placa con agitación (IKAC) modelo C-MAG HS7, North Carolina, USA), durante una hora, a 625 rpm. Para la sonicación se empleó un equipo de ultrasonido (SKYMEN@) DIGITAL ULTRASONIC CLEANER modelo JP-4820, Shenzhen, China), a 35 Khz de frecuencia, durante $64 \mathrm{~min}$. Cada uno de los extractos fue filtrado con bomba de vacío (EVAR@ modelo EV-40, Guadalajara, México) en un embudo de porcelana, usando papel filtro Watman No. 1, para posteriormente ser concentrados en un rotaevaporador (IKA@ modelo RV-10 Digital V, North Carolina, USA), y las recuperaciones fueron almacenadas en viales de vidrio transparente.

\section{Análisis cualitativo de compuestos fitoquímicos}

Las metodologías reportadas por Sofowara (1993), Harborne (1998) y Evans (2009), como técnicas estándar para determinar los compuestos fitoquímicos, fueron utilizadas para determinar cualitativamente la presencia de esteroides, terpenoides, fenoles, taninos, alcaloides, flavonoides, saponinas, antraquinonas y cumarinas de los extractos.

Los esteroides y terpenoides se determinaron al colocar en un tubo de ensaye $150 \mu \mathrm{L}$ del extracto vegetal, donde se le añadieron $1 \mathrm{~mL}$ de cloroformo más $150 \mu \mathrm{L}$ de ácido sulfúrico; una vez que ocurrió la reacción química y que generó un precipitado café-rojizo en el fondo del tubo, se consideró como positiva la prueba, ya que se sugiere la presencia de esteroides; asimismo, la evidencia de una fase café-rojiza en la parte superior indica la presencia de terpenoides (Trease y Evans 1989; Evans 2002). 
Los fenoles y taninos se determinaron al colocar en un tubo de ensaye $150 \mu \mathrm{L}$ de extracto vegetal, al cual se le agregaron $150 \mu \mathrm{L}$ de cloruro férrico $\left(\mathrm{FeCl}_{3}\right)$ a 10 por ciento. Un precipitado de color verde indicó la presencia de fenoles y taninos (Trease y Evans 1989; Evans 2002).

La presencia de alcaloides en las muestras fue determinada al colocar en un tubo de ensaye $150 \mu \mathrm{L}$ de extracto vegetal, más $150 \mu \mathrm{L}$ de ácido clorhídrico $(\mathrm{HCl})$ $2 \mathrm{~N}$ y $150 \mu \mathrm{L}$ de reactivo de Mayer's. Una presencia del precipitado blanco es indicativo a positivo de alcaloides (Trease y Evans 1989; Evans 2002).

Los flavonoides se determinaron de acuerdo con la metodología de Trease y Evans (1989) y Evans (2002); para esto, en un tubo de ensaye se pusieron $150 \mu \mathrm{L}$ de extracto vegetal, para luego agregarle 1 mL de amoníaco al $10 \%$ (en forma de $\mathrm{NH}_{4} \mathrm{OH}$ ), lo que generó una reacción color amarillo; al adicionar $1 \mathrm{~mL}$ de $\mathrm{H}_{2} \mathrm{SO}_{4}$ concentrado, cambió la coloración de amarillo a naranja, lo cual es indicativo de la presencia de flavonoides.

Las antraquinonas fueron determinadas en un tubo de ensaye donde se adicionó $1 \mathrm{~mL}$ de benceno más $1 \mathrm{~mL}$ de amoniaco a 10 por ciento (en forma de $\left.\mathrm{NH}_{4} \mathrm{OH}\right)$ y $150 \mu \mathrm{L}$ de extracto vegetal. La reacción química generó dos fases, la del benceno (fase superior) y amoniaco (fase inferior) de color rosa, indicativo de la presencia de antraquinonas (Trease y Evans 1989; Evans 2002).

La determinación de saponinas se llevó a cabo en un tubo de ensaye, al cual se agregaron $150 \mu \mathrm{L}$ de extracto vegetal y $2 \mathrm{~mL}$ de agua destilada, que posteriormente se agitaron durante $10 \mathrm{~s}$, con ayuda de un agitador (VORTEX-GENIE@ II MIXER, Modelo SI-0236, New York, USA). El resultado es positivo para saponinas una vez que se generen burbujas durante la agitación y que éstas sean persistentes (Trease y Evans 1989; Evans 2002).

El análisis de las acetogeninas se hizo a través de una cromatografía en capa fina de cada uno de los extractos, para evaluar la presencia de acetogeninas revelando con el reactivo de Keede ( $\mathrm{Gu}$ et al. 1995). De manera complementaria, se realizó una prueba en tubo de ensaye, al cual se le agregaron $150 \mu \mathrm{L}$ de extracto vegetal y se le adicionaron $150 \mu \mathrm{L}$ de reactivo A (Ácido 3-5 dinitrobenzoico) y $150 \mu \mathrm{L}$ de reactivo B $(\mathrm{KOH})$; la reacción es positiva para acetogeninas con la generación de un color rosa mexicano. Los resultados fueron expresados con presencia (+) o ausencia (-) del compuesto fitoquímico que se evaluó.

Los fenoles totales se determinaron utilizando la metodología de Maksimović et al. (2005), con modificaciones. Se usó un tubo de ensaye y se añadieron $50 \mu \mathrm{L}$ de extracto vegetal y $250 \mu \mathrm{L}$ de agua destilada, más $125 \mu \mathrm{L}$ de reactivo de Folin-Ciocalteu y 625 $\mu \mathrm{L}$ de una solución de carbonato de calcio a 20 por ciento; los tubos en reacción se dejaron reposar durante $40 \mathrm{~min}$ en la oscuridad. Posteriormente, se tomaron $200 \mu \mathrm{L}$ de la reacción y se colocaron en una microplaca, para ser leídos en un lector de microplacas (THERMO SCIENTIFIC@, modelo: MULTISKAN GO, Massachusetts, USA) a $725 \mathrm{~nm}$. Los datos se calcularon bajo una curva de calibración con una curva de ácido gálico, y los resultados se expresaron en Meq de ácido gálico (EAG)/ g de tejido liofilizado.

Los flavonoides totales se cuantificaron de acuerdo con el método de Maksimović et al. (2005). Para ello, se adicionó $1.4 \mathrm{~mL}$ de agua desionizada y $0.5 \mathrm{~mL}$ del reactivo de flavonoides a $0.1 \mathrm{~mL}$ de extracto; se dejó reposar durante $30 \mathrm{~min}$ en oscuridad y la absorbancia se midió a $430 \mathrm{~nm}$ en un lector de microplacas (THERMO SCIENTIFIC@ ${ }^{\circ}$ modelo: MULTISKAN GO, Massachusetts, USA); el contenido de flavonoides totales se expresó como mg equivalentes de rutina / $\mathrm{g}$ de materia seca (mg ER/g MS).

Los alcaloides totales se mezclaron $2 \mathrm{~g}$ de extracto en $\mathrm{HCl} 2 \mathrm{~N}(1: 1)$ y se filtraron con papel Whatman Núm. 1. Se hicieron tres lavados con cloroformo a 1 mL. Se ajustó el $\mathrm{pH}$ a la neutralidad con $\mathrm{NaOH} 0.1$ $\mathrm{N}$. La solución se depositó dentro de un embudo de separación y se añadieron $5 \mathrm{~mL}$ de solución de verde de bromocresol y $5 \mathrm{~mL}$ de buffer de fosfato a $\mathrm{pH}$ 4.7; se agitó la mezcla y se extrajo el complejo alcaloide-verde de bromocresol con 1, 2, 3 y $4 \mathrm{~mL}$ de cloroformo. Se recuperó el complejo en color amarillo en un matraz volumétrico de $10 \mathrm{~mL}$ y se aforó con cloroformo. Se tomaron $200 \mu \mathrm{L}$ y se depositó en un pocillo de una microplaca. Se midió la absorbancia a $470 \mathrm{~nm}$ en el lector de microplacas (THERMO SCIENTIFICC), modelo: MULTISKAN GO, Massachusetts, USA). Los resultados se expresaron como mg equivalentes de Atropina/ g de tejido liofilizado (mg EA/g E) (Fazel et al. 2010). 


\section{Resultados y Discusión}

En la evaluación cualitativa de los metabolitos presentes en los extractos de epidermis y pulpa de guanábana se obtuvo nulo efecto del hexano en epidermis (Cuadros 1 y 2). En pulpa, se detectó la presencia de acetogeninas y saponinas (Cuadro 1). La extracción por maceración y sonicación con cloroformo determinó que las inflorescencias y epidermis contienen fenoles, taninos y flavonoides. En frutos en desarrollo, 13 a $15 \mathrm{~cm}$ de tamaño, se detectó la presencia de saponinas; mientras que, en la pulpa, en las seis etapas fenológicas se observa la presencia de acetogeninas con la extracción mediante sonicación. La mayoría de los metabolitos fueron extraídos cuando se utilizó etanol, lo cual se atribuye a la polaridad del disolvente utilizado en los dos métodos de extracción.

En los análisis en la epidermis con extracción de etanol, no se observó la presencia de fenoles y taninos en las etapas fenológicas de cepillo a 13-15 cm; asimismo, en las etapas fenológicas desde los 13-15 cm hasta los 25-30 cm no hubo presencia de terpenoides (Cuadro 1). En pulpa de guanábana en las etapas fenológicas se observó la presencia de esteroides, terpenoides, alcaloides, acetogeninas y saponinas; no se percibió la presencia de fenoles, taninos y antraquinonas (Cuadro 2).

Los resultados obtenidos en este estudio coinciden con lo reportado por Manochai et al. (2018), quienes realizaron una extracción con etanol en epidermis de Annona squamosa L., para reportar un elevado contenido de compuestos fenólicos. En otro estudio realizado en hojas de $A$. muricata donde realizaron extracción con etanol y una purificación con hexano $(\mathrm{v} / \mathrm{v})$, cloruro de metileno, acetato de etilo, n-butanol y agua, se determinó que el menor rendimiento de fenoles totales, flavonoides y protoantocianidinas fue con hexano; presumiblemente, la baja extracción se puede deber a la polaridad del hexano (Justino et al. 2018). Asimismo, De Silva et al. (2017) reportaron que el hexano fue el único disolvente con el cual no se extraen flavonoides en hojas de Annona vepretorum Mart. Existen estudios realizados en pulpa (madurez de consumo) donde se detectó la presencia de acetogeninas extraídas con sonicación y cloroformo (George et al. 2015), lo cual es similar a los resultados obtenidos en esta investigación.
Womeni et al. (2016) reportaron una mayor extracción de compuestos fenólicos con butanol, por lo que concluyeron que la cantidad de compuestos fenólicos es mayor en inflorescencias que en hojas de guanábana; sin embargo, la concentración de fenólicos depende de las condiciones ambientales de desarrollo de la planta y los métodos de extracción.

Pereira et al. (2017) hicieron una extracción por maceración, en epidermis de Annona crasiflora Mart, usando como disolvente etanol a 98 por ciento; posteriormente, hicieron fraccionamiento del extracto con la finalidad de purificar, luego de lo cual encontraron la presencia de un alcaloide staphalagina usado para la inhibición de la enzima lipasa pancreática.

Los resultados en la primera fase mostraron que el metanol es el solvente que posee un mayor espectro de extracción respecto a los demás solventes usados en las inflorescencias y en las fases de crecimiento y desarrollo del fruto de guanábana, lo cual coincide con lo reportado por Agu y Okolie (2017), quienes llevaron a cabo una extracción con metanol de compuestos fitoquímicos en pulpa, hoja, corteza de raíz y corteza de tallo de guanábana.

En la segunda fase, el método de extracción para los fenoles totales en la pulpa del fruto (Figura 1) fue indistinto, ya que no se observaron diferencias estadísticas. En la epidermis se notaron diferencias estadísticas significativas $(P \leq 0.05)$ con los dos métodos de extracción; se obtuvo un mayor contenido de compuestos fenólicos extraídos mediante sonicación. Esto puede atribuirse a la polaridad de los compuestos fenólicos, lo que facilita la extracción con el metanol, aunado a las propiedades de extracción de la sonicación como la cavitación, ya que la energía de implosión

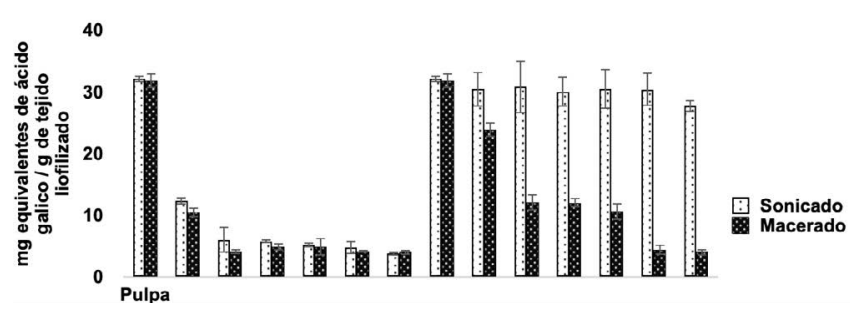

Figura 1. Contenido de fenoles totales en extractos metanólicos de pulpa y epidermis de guanábana, extraídos por sonicación y macerado. Medias de los tratamientos con letras iguales no son estadísticamente diferentes (DMS $=8.10$ ). 
Cuadro 1. Análisis cualitativo de extractos por maceración y sonicación en epidermis de guanábana (Annona muricata L.) en diferentes etapas fenológicas. Inflor: Inflorescencia, Cep: Cepillo, M: macerado, S: sonicado,

Presencia $(+)$, ausencia (-).

\begin{tabular}{|c|c|c|c|c|c|c|c|c|c|c|c|c|c|c|}
\hline \multirow[b]{2}{*}{ Fitoquímico } & \multicolumn{2}{|c|}{ Inflor } & \multicolumn{2}{|c|}{ Cep } & \multicolumn{2}{|c|}{$5-7 \mathrm{~cm}$} & \multicolumn{2}{|c|}{$8-10 \mathrm{~cm}$} & \multicolumn{2}{|c|}{$13-15 \mathrm{~cm}$} & \multicolumn{2}{|c|}{$17-23 \mathrm{~cm}$} & \multicolumn{2}{|c|}{$25-30 \mathrm{~cm}$} \\
\hline & $\mathrm{M}$ & S & M & S & M & S & M & S & $\mathrm{M}$ & $S$ & M & $\mathrm{S}$ & M & S \\
\hline & \multicolumn{14}{|c|}{ Hexano } \\
\hline Esteroides & - & - & - & - & - & - & - & - & - & - & - & - & - & - \\
\hline Terpenoides & - & - & - & - & - & - & - & - & - & - & - & - & - & - \\
\hline Fenoles y taninos & - & - & - & - & - & - & - & - & - & - & - & - & - & - \\
\hline Alcaloides & - & - & - & - & - & - & - & - & - & - & - & - & - & - \\
\hline Acetogeninas & - & - & - & - & - & - & - & - & - & - & - & - & - & - \\
\hline Flavonoides & - & - & - & - & - & - & - & - & - & - & - & - & - & - \\
\hline Antraquinonas & - & - & - & - & - & - & - & - & - & - & - & - & - & - \\
\hline Saponinas & - & - & - & - & - & - & - & - & - & - & - & - & - & - \\
\hline Esteroides & - & - & - & - & - & - & - & - & - & - & - & - & - & - \\
\hline \multirow[t]{2}{*}{ Terpenoides } & - & - & - & - & - & - & - & - & - & - & - & - & - & - \\
\hline & \multicolumn{14}{|c|}{ Cloroformo } \\
\hline Fenoles y taninos & - & - & - & - & - & - & - & - & - & - & - & - & - & - \\
\hline Alcaloides & - & - & - & + & - & - & - & - & - & - & - & - & - & - \\
\hline Acetogeninas & + & + & + & + & + & + & - & - & + & + & + & + & + & + \\
\hline Flavonoides & - & - & - & - & - & - & - & - & - & - & - & - & - & - \\
\hline Antraquinonas & - & - & - & - & - & - & - & - & - & - & - & - & - & - \\
\hline Saponinas & + & + & + & + & - & - & + & + & + & + & + & + & - & + \\
\hline Esteroides & - & - & - & - & - & - & - & - & - & - & - & - & - & - \\
\hline \multirow[t]{2}{*}{ Terpenoides } & - & - & - & - & + & + & + & + & - & - & - & - & - & - \\
\hline & \multicolumn{14}{|c|}{ Etanol } \\
\hline Fenoles y taninos & + & + & - & - & - & - & - & - & - & + & + & + & - & - \\
\hline Alcaloides & + & + & + & + & + & + & + & + & - & + & + & + & - & - \\
\hline Acetogeninas & + & + & + & + & + & + & + & + & + & + & + & + & + & + \\
\hline Flavonoides & + & + & + & + & + & + & + & + & + & + & + & + & + & + \\
\hline Antraquinonas & + & + & + & + & + & + & + & + & + & + & + & + & + & + \\
\hline Saponinas & + & + & + & + & + & + & + & + & + & + & + & + & + & + \\
\hline Esteroides & + & + & + & + & + & + & + & + & + & + & + & + & + & + \\
\hline \multirow[t]{2}{*}{ Terpenoides } & + & + & - & - & + & + & + & + & - & - & - & - & - & - \\
\hline & \multicolumn{14}{|c|}{ Metanol } \\
\hline Fenoles y taninos & + & + & + & + & + & + & + & + & + & + & + & + & + & + \\
\hline Alcaloides & + & + & + & + & + & + & + & + & + & + & + & + & + & + \\
\hline Acetogeninas & + & + & + & + & + & + & + & + & + & + & + & + & + & + \\
\hline Flavonoides & + & + & + & + & + & + & + & + & + & + & + & + & + & + \\
\hline Antraquinonas & + & + & + & + & + & + & + & + & + & + & + & + & + & + \\
\hline Saponinas & + & + & + & + & + & + & - & - & + & + & + & + & + & + \\
\hline
\end{tabular}


Cuadro 2. Análisis cualitativo de extractos por maceración y sonicación en pulpa de guanábana (Annona muricata L.) en diferentes etapas fenológicas. Inflor: Inflorescencia, Cep: Cepillo, M: macerado, S: sonicado, Presencia (+), ausencia (-).

\begin{tabular}{|c|c|c|c|c|c|c|c|c|c|c|c|c|c|c|}
\hline \multirow[b]{2}{*}{ Fitoquímico } & \multicolumn{2}{|c|}{ Inflor } & \multicolumn{2}{|c|}{ Cep } & \multicolumn{2}{|c|}{$5-7 \mathrm{~cm}$} & \multicolumn{2}{|c|}{$8-10 \mathrm{~cm}$} & \multicolumn{2}{|c|}{$13-15 \mathrm{~cm}$} & \multicolumn{2}{|c|}{$17-23 \mathrm{~cm}$} & \multicolumn{2}{|c|}{$25-30 \mathrm{~cm}$} \\
\hline & $\mathrm{M}$ & $S$ & $\mathrm{M}$ & $S$ & $\mathrm{M}$ & $S$ & $\mathrm{M}$ & $S$ & $\mathrm{M}$ & $S$ & $\mathrm{M}$ & $S$ & M & $S$ \\
\hline & \multicolumn{14}{|c|}{ Hexano } \\
\hline Esteroides & - & - & - & - & - & - & - & - & - & - & - & - & - & - \\
\hline Terpenoides & - & - & - & - & - & - & - & - & - & - & - & - & - & - \\
\hline Fenoles y taninos & - & - & - & - & - & - & - & - & - & - & - & - & - & - \\
\hline Alcaloides & - & - & - & - & - & - & - & - & - & - & - & - & - & - \\
\hline Acetogeninas & - & - & - & - & - & + & - & + & - & + & - & + & - & - \\
\hline Flavonoides & - & - & - & - & - & - & - & - & - & - & - & - & - & - \\
\hline Antraquinonas & - & - & - & - & - & - & - & - & - & - & - & - & - & - \\
\hline Saponinas & - & - & + & + & - & - & - & - & + & + & + & + & - & - \\
\hline Esteroides & - & - & - & - & - & + & - & - & - & - & + & + & - & - \\
\hline \multirow[t]{2}{*}{ Terpenoides } & - & - & - & - & - & + & - & - & - & - & + & + & - & - \\
\hline & \multicolumn{14}{|c|}{ Cloroformo } \\
\hline Fenoles y taninos & + & + & - & - & - & - & - & - & - & - & - & - & - & - \\
\hline Alcaloides & - & - & - & - & - & - & - & - & - & - & - & - & - & - \\
\hline Acetogeninas & - & - & + & + & + & + & + & + & + & + & + & + & + & + \\
\hline Flavonoides & + & + & - & - & - & - & - & - & - & - & - & - & - & - \\
\hline Antraquinonas & - & - & - & - & - & - & - & - & - & - & - & - & - & - \\
\hline Saponinas & - & - & + & + & - & + & - & + & - & - & + & - & - & - \\
\hline Esteroides & + & + & + & + & + & - & - & + & + & + & + & + & - & + \\
\hline \multirow[t]{2}{*}{ Terpenoides } & + & + & + & + & + & - & - & + & + & + & + & + & - & + \\
\hline & \multicolumn{14}{|c|}{ Etanol } \\
\hline Fenoles y taninos & + & + & - & - & - & - & - & - & - & - & - & - & - & - \\
\hline Alcaloides & + & + & + & + & + & + & + & + & + & + & + & + & + & + \\
\hline Acetogeninas & + & + & - & + & - & + & - & + & - & + & - & + & - & - \\
\hline Flavonoides & + & + & + & + & + & + & + & + & + & + & + & + & + & + \\
\hline Antraquinonas & + & + & - & - & - & - & - & - & - & - & - & - & - & - \\
\hline Saponinas & + & + & - & - & + & + & + & + & + & + & - & - & - & - \\
\hline Esteroides & + & + & + & + & + & + & - & + & + & + & + & + & + & + \\
\hline \multirow[t]{2}{*}{ Terpenoides } & + & + & + & + & + & + & - & + & + & + & + & + & + & + \\
\hline & \multicolumn{14}{|c|}{ Metanol } \\
\hline Fenoles y taninos & + & + & + & + & + & - & + & + & + & + & + & + & - & - \\
\hline Alcaloides & + & + & + & + & + & + & + & + & + & + & + & + & + & + \\
\hline Acetogeninas & + & + & + & + & + & + & + & + & + & + & + & + & - & - \\
\hline Flavonoides & + & + & - & - & + & + & + & + & + & + & + & + & + & + \\
\hline Antraquinonas & + & + & - & - & - & - & - & - & - & - & - & - & - & - \\
\hline Saponinas & - & - & - & - & - & - & - & - & - & - & + & + & + & + \\
\hline
\end{tabular}


liberada favorece la ruptura celular y la liberación del contenido intracelular (He et al. 2016).

En el contenido de alcaloides totales en pulpa se observaron diferencias estadísticas $(P \leq 0.05)$ con los dos métodos de extracción, a partir del fruto de $8-10 \mathrm{~cm}$ hasta $25-30 \mathrm{~cm}$ de longitud, pero fue con el sonicado con el que se extrajo el mayor contenido (Figura 2), con un incremento desde la inflorescencia (153.3 mg equivalentes de atropina/g de extracto) hasta la fase donde el fruto era de $17-23 \mathrm{~cm}$ de longitud (325.25 mg equivalentes de atropina/g de extracto) y un decremento en la madurez de consumo (173.75 mg equivalentes de atropina/g de extracto). Al respecto, Womeni et al. (2016) reportaron que los alcaloides poseen propiedades de defensa en frutos, mismos que son exigidos durante las diversas etapas fenológicas de éste; asimismo, se han reportado alcaloides del tipo aporfinos e isoquinol, en pulpa y cáscara de guanábana (Fofana et al. 2011; Fofana et al. 2012).

En la pulpa, se extrajeron más alcaloides en la fase del fruto de 5-7 cm y 13-15 cm de longitud, con respecto a la epidermis en la misma fase (Figura 2). Aguilar-Hernández et al. (2020) reportaron un contenido de alcaloides totales extraídos por ultrasonido en pulpa $(1.09 \mathrm{mg} / \mathrm{g})$, semilla $(2.02 \mathrm{mg} / \mathrm{g})$, columnela $(0.83 \mathrm{mg} / \mathrm{g})$ y epidermis $(7.32 \mathrm{mg} / \mathrm{g})$ de guanábana, gracias a lo cual determinaron que la complejidad del órgano estructural vegetal y la etapa fenológica son factores importantes en la extracción de alcaloides. El incremento en el contenido de alcaloides en las plantas se relaciona con

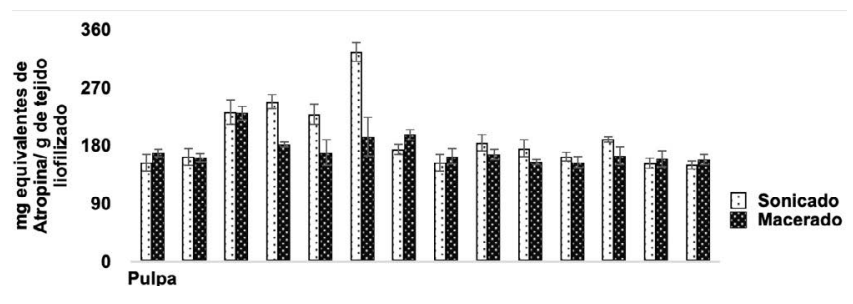

Figura 2. Contenido de alcaloides totales en extractos metanólicos de pulpa y epidermis de guanábana, extraídos por sonicación y macerado. Medias de los tratamientos con letras iguales no son estadísticamente diferentes (DMS $=21.41$ ).

su actividad tóxica en respuesta a la interacción de insectos y herbívoros con las plantas (Macel et al. 2005).

La mayor concentración de contenido deflavonoides (Figura 3) se obtuvo en las inflorescencias de guanábana por el método de extracción por sonicación $(1,060 \mathrm{mg} / \mathrm{g}$ de extracto liofilizado), y se observaron diferencias es- tadísticas $(P \leq 0.05)$ respecto al macerado $(490.83 \mathrm{mg} / \mathrm{g}$ de extracto liofilizado); estas diferencias pueden atribuirse a la eficacia de extracción de la sonicación (He et al. 2016).

En la pulpa del fruto, con los dos métodos de extracción, a partir de la fase donde los frutos presentaban una longitud de 13-15, 17-25 y $25-30 \mathrm{~cm}$, se observaron diferencias estadísticas $(P \leq 0.05)$; comportamiento similar se observó en la epidermis en el contenido de flavonoides (dos métodos de extracción) en las fases del fruto de los 5-7 hasta los $25-30 \mathrm{~cm}$ de longitud. Así, la mayor concentración de flavonoides totales se cuantificó en las inflorescencias de guanábana por sonicado y macerado. Se ha reportado que la factibilidad de extracción de compuestos polifenólicos va a depender de qué tan ligados se encuentren a ma-

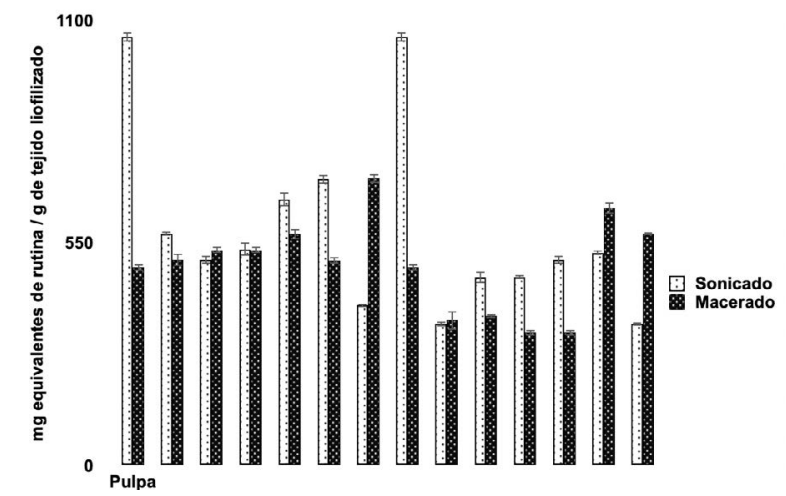

Figura 3. Contenido de flavonoides totales en extractos metanólicos de pulpa y epidermis de guanábana, extraídos por sonicación y macerado. Medias de los tratamientos con letras iguales no son estadísticamente diferentes $(\mathrm{DMS}=121.58)$.

cromoléculas de otras partes de la planta, por lo que son más fáciles de solubilizar (Evergetis y Haroutounian 2014). Los flavonoides actúan como mecanismos de defensa ante herbívoros, en los sépalos, que son parte de la inflorescencia; éstos confieren una acción protectora de los órganos reproductores; de igual forma, flavonoides tienden a disminuir a lo largo del desarrollo del fruto (Vargas-Alvares et al. 2006).

\section{Conclusión}

Extracciones mediante el método por sonicado, utilizando metanol, obtuvieron un alto contenido de compuestos bioactivos de los órganos estructurales, en las etapas fenológicas durante el crecimiento y desarrollo de la guanábana. Las concentraciones más 
elevadas de compuestos fenólicos se encontraron en inflorescencias y epidermis de los frutos; en la pulpa, alto contenido de flavonoides y alcaloides. Estos compuestos bioactivos tienen propiedades anticancerígenas, cicatrizantes y antioxidantes, por lo cual es muy importante la determinación del contenido de éstos en guanábana para realizar estudios posteriores.

\section{Agradecimientos}

El proyecto fue apoyado por el Fondo Sectorial para la Educación (CONACyT-SEP) número 242718 y Fondo Sectorial para la Investigación en Materias Agrícola, Pecuaria, Acuacultura, Agrobiotecnología y Recursos Fitogenéticos (CONACyT-SADER) número de subvención 266891. 


\section{Literatura Citada}

Abarca-Vargas R, Petricevich VL. 2018. Importancia biológica de los compuestos fenólicos. Inventio 14: 33-38. 10.30973/INVENTIO/2018.14.34/4

Agu KC, Okolie PN. 2017. Proximate composition, phytochemical analysis, and in vitro antioxidant potentials of extracts of Annona muricata (Soursop). Food Science \& Nutrition 5: 1029-1036. https://doi.org/10.1002/fsn3.498

Agu KC, Okolie NP, Falodun A, Engel-Lutz N. 2018. In vitro anticancer assessments of Annona muricata fractions and in vitro antioxidant profile of fractions and isolated acetogenin (15-acetyl guanacone). Journal of Cancer Research and Practice 5: 53-66. https://doi.org/10.1016/j. jcrpr.2017.12.001

Aguilar-Hernández G, Zepeda-Vallejo LG, García-Magaña MdeL, Vivar-Vera MdelosÁ, Pérez-Larios A, GirónPérez MI, Montalvo-González E. 2020. Extraction of alkaloids using ultrasound from pulp and by-Products of soursop fruit (Annona muricata L.). Applied Sciences 10: 1-15. https://doi.org/10.3390/app10144869

Ávalos-García A, Pérez-Urria CE. 2009. Metabolismo secundario de plantas. Reduca (Biología). Serie Fisiología Vegetal 2: 119-145.

Badrie N, Schauss AG. 2010. Soursop (Annona muricata L.): Composition, nutritional value, medicinal uses, and toxicology. En: Ross R, Preedy V, editores. Bioactive Foods in Promoting Health. San Diego, Academic Press. P. 621-643. https://doi.org/10.1016/ B978-0-12-374628-3.00039-6

Broun P, Liu Y, Queen E, Schwarz Y, Abenes ML, Leibman M. 2006. Importance of transcription factors in the regulation of plant secondary metabolism and their relevance to the control of terpenoid accumulation. Phytochemistry Reviews 5: 27-38. https://doi. org/10.1007/s11101-006-9000-x

Cabrera-Carrión JL, Jaramillo-Jaramillo C, Dután-Torres F, Cun-Carrión J, García PA, Rojas de Astudillo L. 2017. Variación del contenido de alcaloides, fenoles, flavonoides y taninos en Moringa oleifera Lam. en función de su edad y altura. Bioagro 29: 53-60.

Champy P, Melot A, Guérineau-Eng V, Gleye C, Fall D, Höglinger GU, Rugber M, Lannuzel A, Laprévote O, Laurens A, Hocquemiller R. 2005. Quantification of acetogenins in Annona muricata linked to atypical parkinsonism in Guadeloupe. Movement Disorders 20: 16291633. https://doi.org/10.1002/mds.20632
[CONAGUA] Comisión Nacional del Agua. [internet]. 2017. Información climatológica 2017. [citado 2021 mayo 21]. Disponible en: https://smn.conagua. gob.mx/es/climatologia/informacion-climatologica/ informacion-estadistica-climatologica.

Cordeiro MCR, Pinto ACdeQ. 2005. Properties. En: Williams JT, Smith RW, Huhes A, Haq N, Clement CR, editores. Annona Species. Southampton, International Centre for Underutilised Crops. P. 33-38.

Coria-Téllez AV, Montalvo-Gónzalez E, Yahia EM, ObledoVázquez EN. 2018. Annona muricata: A comprehensive review on its traditional medicinal uses, phytochemicals, pharmacological activities, mechanisms of action and toxicity. Arabian Journal of Chemistry 11: 662-691. https://doi.org/10.1016/j.arabjc.2016.01.004

Correa-Gordillo J, Ortiz D, Larrahondo JE, Sánchez-Mejía M, Pachon H. 2012. Actividad antioxidante en guanábana (Annona muricata L.): una revisión bibliográfica. Boletín Latinoamericano y del Caribe de Plantas Medicinales y Aromáticas 11: 111-126.

Evans WC. 2002. Trease and Evan's Pharmacognosy. 15th edition. Haarcourt Brace and Company. Reino Unido.

Evans WC. 2009. Trease and Evan's Pharmacognosy. 16th edition. Elsevier Health Sciences. Reino Unido.

Evergetis E, Haroutounian SA. 2014. Exploitation of Apiaceae family plants as valuable renewable source of essential oils containing crops for the production of fine chemicals. Industrial Crops and Products 54: 7077. https://doi.org/10.1016/j.indcrop.2014.01.009

Fazel S, Hamidreza M, Rouhollah G, Verdian-Rizi M. 2010. Spectrophotometric determination of total alkaloids in some Iranian medicinal plants. Journal of Applied Horticulture 12: 69-70. https://doi.org/10.37855/ JAH.2010.V12I01.15

Fofana S, Ziyaev R, Abdusamatov A, Zakirov SK. 2011. Alkaloids from Annona muricata leaves. Chemistry of Natural Compounds 47: 321-321. https:/doi. org/10.1007/s10600-012-0363-5

Fofana S, Keita A, Balde S, Ziyaev R, Aripova SF. 2012. Alkaloids from leaves of Annona muricata. Chemistry of Natural Compounds 48: 714-714. https:/doi. org/10.1007/s10600-012-0363-5

George VC, Kumar DN, Suresh PK, Kumar RA. 2015. Antioxidant, DNA protective efficacy and HPLC analysis of Annona muricata (soursop) extracts. Journal of Food Science and Technology 52: 2328-2335. https:// doi.org/10.1007/s13197-014-1289-7 
Gu ZM, Zhao GX, Oberlies NH, Zeng L, McLaughlin JL. 1995. Annonaceous acetogenins. En: Arnason JT, Mata R, Romeo JT, editores. Phytochemistry of Medicinal Plants. Boston, Springer Press. P. 249-310. https://doi. org/10.1007/978-1-4899-1778-2_11

Harborne JB. 1998. Photochemical Methods: A Guide to Modern Techniques of Plant Analysis. Third edition. Chapman \& Hall. Reino Unido.

Hasrat JA, Pieters L, De Backer JP, Vauquelin G, Vlietinck AJ. 1997a. Screening of medicinal plants from Suriname for 5-HT 1A ligands: Bioactive isoquinoline alkaloids from fruit of Annona muricata. Phytomedicine 4: 133140. https://doi.org/10.1016/S0944-7113(97)80059-1

Hasrat JA, De Bruyne T, De Backer JP, Vauquelin G, Vlietinck AJ. 1997b. Isoquinoline derivatives isolated from the fruit of Annona muricata as 5-HTergic 5-HT1A receptor agonists in rats: Unexploited antidepressive (lead) products. Journal of Pharmacy and Pharmacology 49: 1145-1149. https://doi. org/10.1111/j.2042-7158.1997.tb06058.x

He B, Zhang LL, Yue XY, Liang J, Jiang J, Gao XL, Yue PX. 2016. Optimization of ultrasound-assisted extraction of phenolic compounds and anthocyanins from blueberry (Vaccinium ashei) wine pomace. Food Chemistry 204: 70-76. https://doi.org/10.1016/j.foodchem.2016.02.094

Hernández-Fuentes GA, García-Argáez AN, PerazaCampos AL, Delgado-Enciso I, Muñiz-Valencia R, Martínez-Martínez FJ, Toniello A, Gómez-Sandoval Z, Mojica-Sánchez JP, Dalla-Via L, Parra-Delgado H. 2019. Cytotoxic acetogenins from the roots of Annona purpurea. International Journal of Molecular Science 20: 2-24. https://doi.org/10.3390/ijms20081870

[INEGI] Instituto Nacional de Estadística y Geografía. [internet]. 2017. Espacio y datos de México 2017. [citado 2021 mayo 21]. Disponible en: https://www. inegi.org.mx/app/mapa/espacioydatos/default. aspx?ag=180080003.

Jiménez VM, Gruschwitz M, Schweiggert RM, Carle R, Esquivel P. 2014. Identification of phenolic compounds in soursop (Annona muricata) pulp by high-performance liquid chromatography with diode array and electrospray ionization mass spectrometric detection. Food Research International 65: 42-46. https://doi. org/10.1016/j.foodres.2014.05.051

Jiménez-Zurita JO, Balois-Morales R, Alia I, Juárez P, Sumaya MT, Bello JE. 2016. Caracterización de frutos de guanábana (Annona muricata L.) en Tepic, Nayarit,
México. Revista Mexicana de Ciencias Agrícolas 7: 1261-1270. https://doi.org/10.29312/remexca.v7i6.175

Justino AB, Miranda NC, Franco RR, Martins MM, Da Silva NM, Espindola FS. 2018. Annona muricata Linn. leaf as a source of antioxidant compounds with in vitro antidiabetic and inhibitory potential against $\alpha$-amylase, $\alpha$-glucosidase, lipase, non-enzymatic glycation and lipid peroxidation. Biomedicine \& Pharmacotherapy 100: 83-92. https://doi.org/10.1016/j.biopha.2018.01.172 Kotake K, Okuda M, Kamizono N, Matsumoto T, Tanahashi H, Hara H, Ohta S. 2004. Detection and determination of reticuline and $\mathrm{N}$-methylcoculaurine in the Annonaceae family using liquid chromatography-tandem mass spectrometry. Journal of Chromatography B 806: 75-78. https://doi.org/10.1016/j. jchromb.2004.03.017

León AE, Obledo EN, Vivar MdelosA, Ayerdi SGS, Montalvo E. 2017. Evaluation of emerging methods on the polyphenol content, antioxidant capacity and qualitative presence of acetogenins in soursop Pulp (Annona muricata L.). Revista Brasileira de Fruticultura 39: 1-8. https://doi.org/10.1590/0100-29452017358

Macel M, Bruinsma M, Dijkstra SM, Ooijendijk T, Niemeyer HM, Klinkhamer PG. 2005. Differences in effects of pyrrolizidine alkaloids on five generalist insect herbivore species. Journal of Chemical Ecology 31: 1493-1508. https://doi.org/10.1007/s10886-005-5793-0

MaksimovićZ, Malenčić Đ, Kovačević N. 2005. Polyphenol contents and antioxidant activity of Maydis stigma extracts. Bioresourse Technology 96: 873-877. https://doi. org/10.1016/j.biortech.2004.09.006

Manochai B, Ingkasupart P, Lee SH, Hong JH. 2018. Evaluation of antioxidant activities, total phenolic content (TPC), and total catechin content (TCC) of 10 sugar apple (Annona squamosa L.) cultivar peels grown in Thailand. Food Science and Technology 38: 294-300. https://doi.org/10.1590/fst.22117

Melot A, Fall D, Gleye C, Champy P. 2009. Apolar Annonaceous acetogenins from the fruit pulp of Annona muricata. Molecules 14: 4387-4395. https://doi. org/10.3390/molecules14114387

Moghadamtousi S, Fadaeinasab M, Nikzad S, Mohan G, Ali H, Kadir H. 2015. Annona muricata (Annonaceae): A review of its traditional uses, isolated acetogenins and biological activities. International Journal of Molecular Sciences 16: 15625-15658. https://doi. org/10.3390/ijms160715625 
Mohanty S, Hollinshead J, Jones L, Jones PW, Thomas D, Watson AA, Watson DG, Gray AI, Molyneux RJ, Nash RJ. 2008. Annona muricata (Graviola): Toxic or therapeutic. Natural Product Communications 2: 31-33. https://doi.org/10.1177/1934578X0800300107

Nawwar M, Ayoub N, Hussein S, Hashim A, El-Sharawy R, Wende K, Lindequist U. 2012. Flavonol triglycoside and investigation of the antioxidant and cell stimulating activities of Annona muricata Linn. Archives of Pharmacal Research 35: 761-767. https://doi. org/10.1007/s12272-012-0501-4

Pereira MN, Justino AB, Martins MM, Peixoto LG, Vilela DD, Santos PS, Espindola FS. 2017. Stephalagine, an alkaloid with pancreatic lipase inhibitory activity isolated from the fruit peel of Annona crassiflora Mart. Industrial Crops and Products 97: 324-329. https://doi. org/10.1016/j.indcrop.2016.12.038

RupprechtJK, Hui YH, McLaughlinJL. 1990. Annonaceous acetogenins: A review. Journal of Natural Products 53: 237-278. https://doi.org/10.1021/np50068a001

Silva MG, De Oliveira AP, S Araújo C, De Lavor ÉM, Silva JC, Mendes RL, O Pessoa C, P Costa M, Da S Almeida JR. 2017. Phytochemical screening, cytotoxicity and acute toxicity of Annona vepretorum Mart (Annonaceae) leaf extracts. Tropical Journal of Pharmaceutical Research 16: 597-604. https://doi. org/10.4314/tjpr.v16i3.14

Sofowara EA. 1993. Medicinal Plants and Traditional Medicine in Africa. Spectrum Books Ltd. Nigeria.

Sun S, Liu J, Kadouh H, Sun X, Zhou K. 2014. Three new anti-proliferative Annonaceous acetogenins with mono-tetrahydrofuran ring from graviola fruit (Annona muricata). Bioorganic \& Medicinal Chemistry Letters 24: 2773-2776. https://doi.org/10.1016/j. bmcl.2014.03.099

Trease GE, Evans WC. 1989. Pharmacognosy. 13th edition. Bailliere Tindall. Reino Unido.

Vargas-Alvarez D, Soto-Hernández M, GonzálezHernández VA, Mark-Engleman E, Martínez-Garza Á. 2006. Cinética de acumulación y distribución de flavonoides en guayaba (Psidium guajava L.). Agrociencia 40: 109-115.

Verma N, Shukla S. 2015. Impact of various factors responsible for fluctuation in plant secondary metabolites. Journal of Applied Research on Medicinal and Aromatic Plants 2: 105-113. https://doi.org/10.1016/j. jarmap.2015.09.002
Womeni HM, Tonfack-Djikeng F, Iruku NSSP, Karuna MSL, Prasad RBN, Linder M. 2016. Valorization of soursop flowers (Annona muricata L.) as potent source of natural antioxidants for stabilization of palm olein during accelerated storage. Food Science and Nutrition 4: 802-810. https://doi.org/10.1002/fsn3.349 Yang C, Gundala SR, Mukkavilli R, Vangala S, Reid MD, Aneja R. 2015. Synergistic interactions among flavonoids and acetogenins in graviola (Annona muricata) leaves confer protection against prostate cancer. Carcinogenesis 36: 656-665. https://doi.org/10.1093/ carcin/bgv046 